\title{
Author Correction: Direct observation of excitonic instability in $\mathrm{Ta}_{2} \mathrm{NiSe}_{5}$
}

Kwangrae Kim, Hoon Kim, Jonghwan Kim, Changil Kwon (1), Jun Sung Kim \& B. J. Kim (1)

Correction to: Nature Communications https://doi.org/10.1038/s41467-021-22133-z, published online 30 March 2021.

The original version of this Article contained an error in Fig. 1, in which the data was not corrected for the Bose factor.

The correct version is:



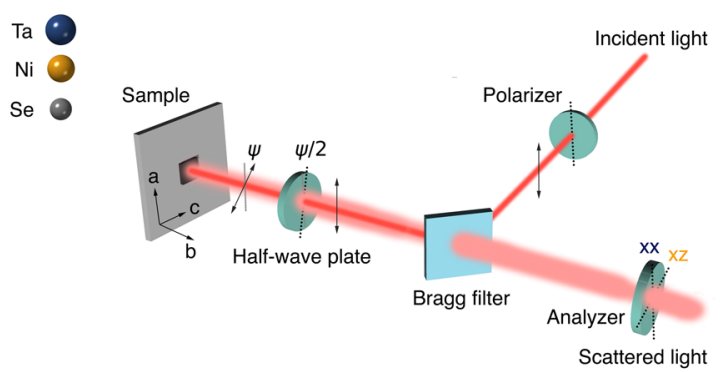

e

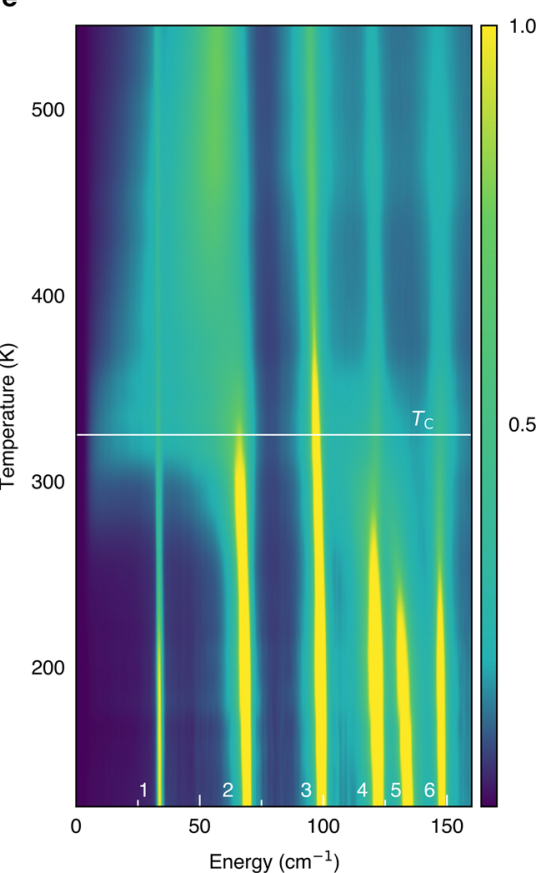


which replaces the previous incorrect version:

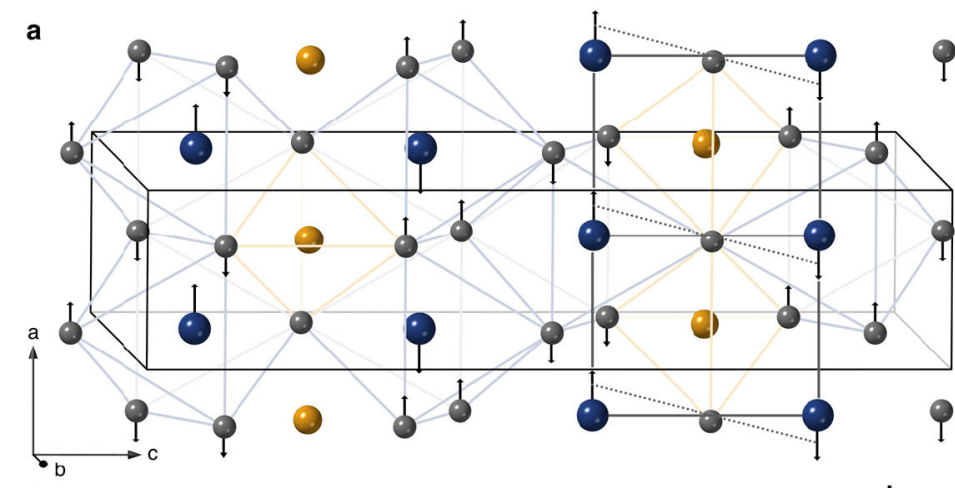

c



b

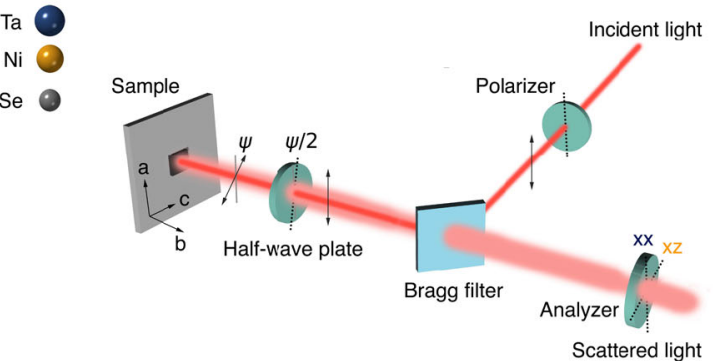

e
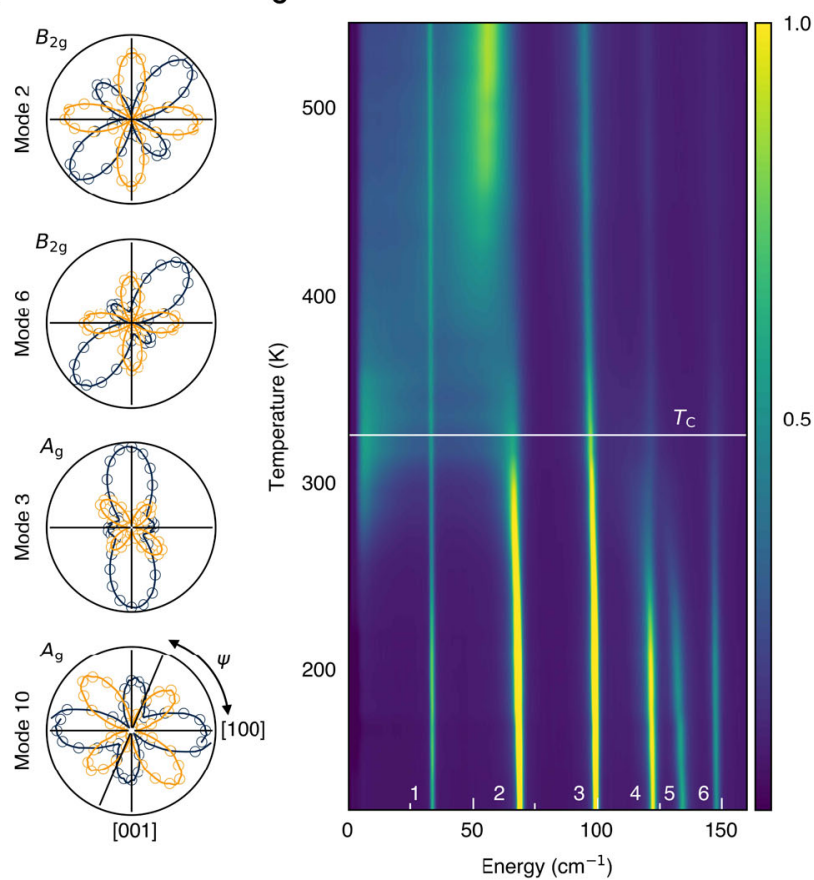
The original version of this Article contained an error in Fig. 2, in which the data was not corrected for the Bose factor.

The correct version is:
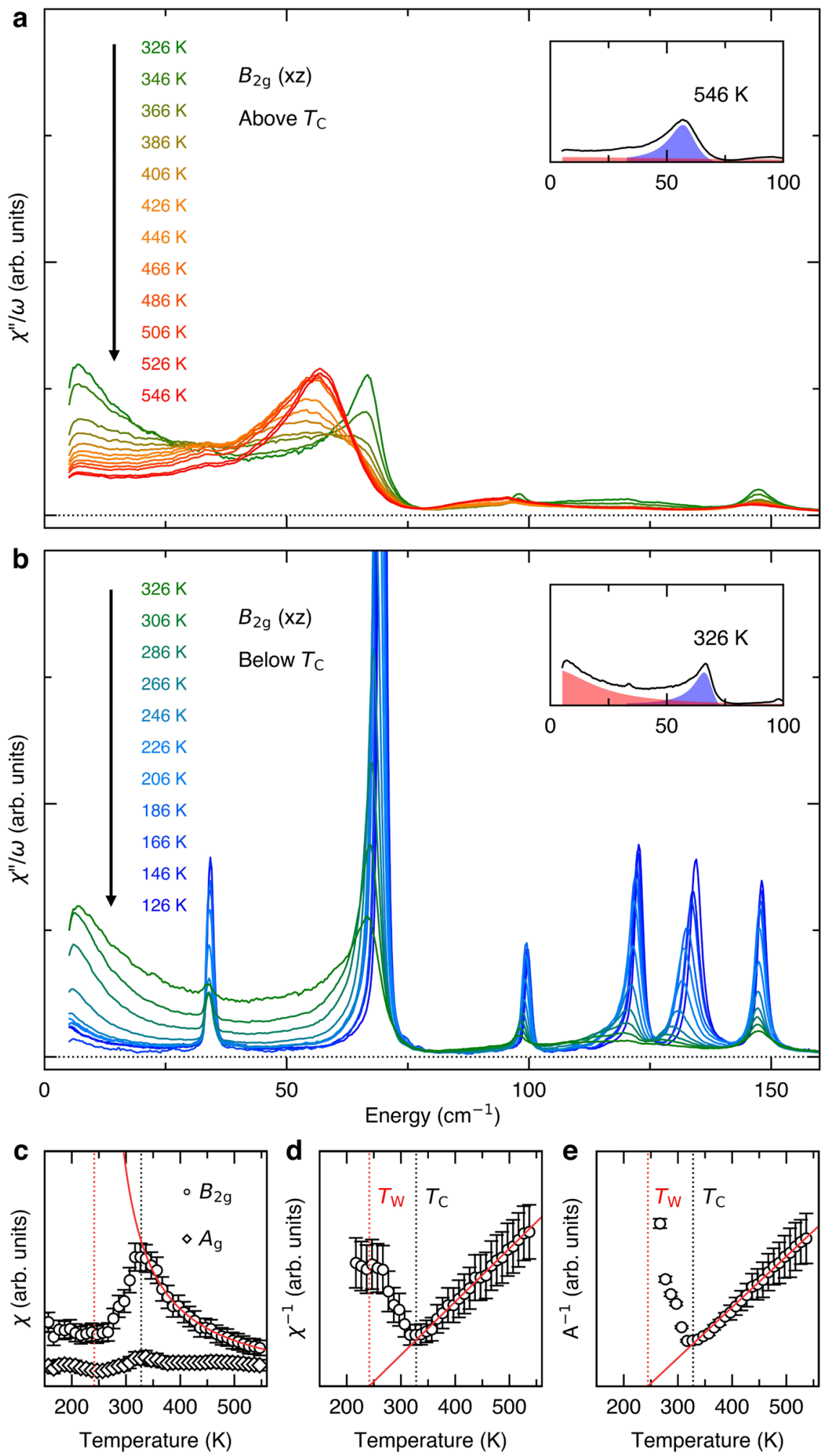
which replaces the previous incorrect version:
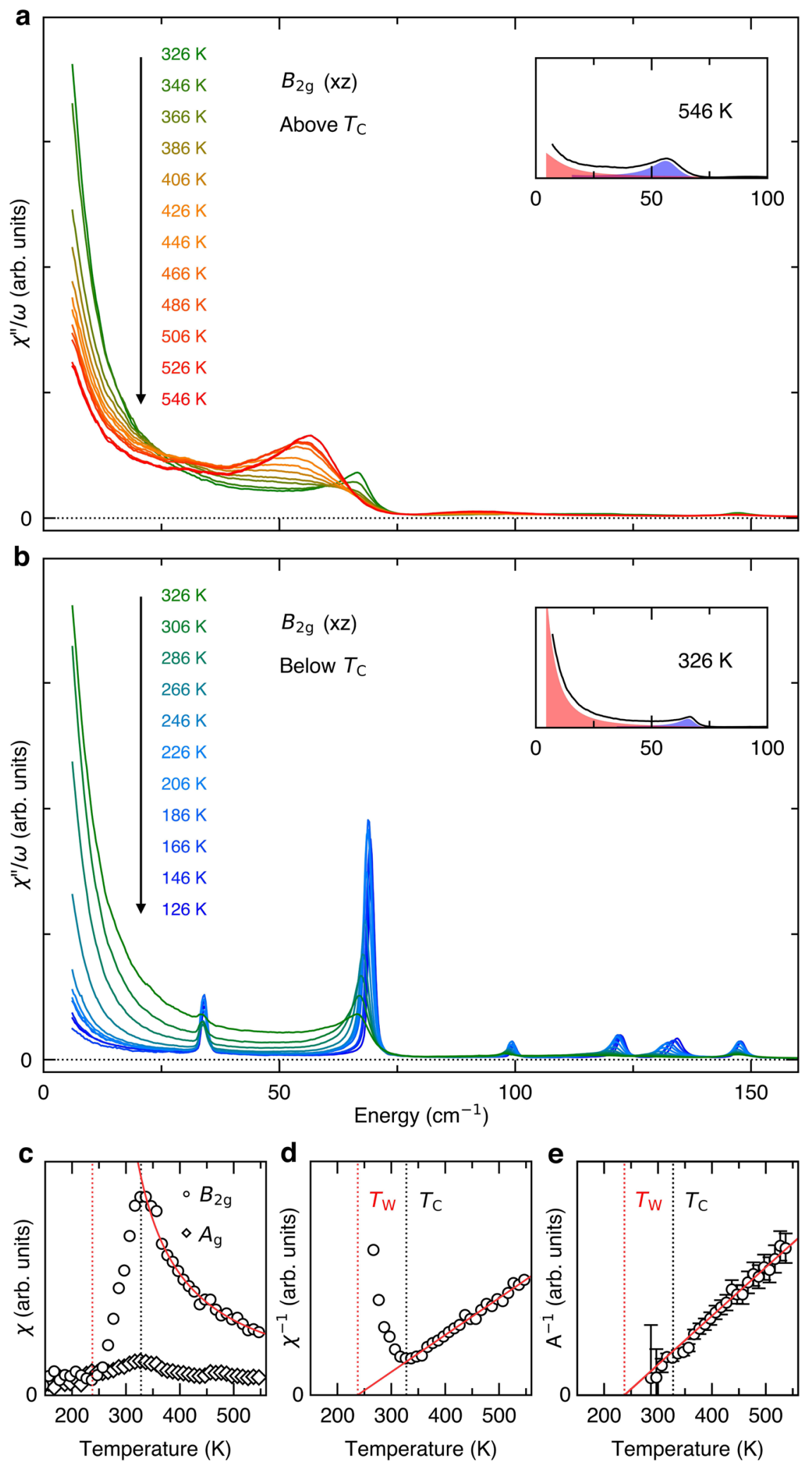
The original version of this Article contained an error in Fig. 3, in which the data was not corrected for the Bose factor.

The correct version is:
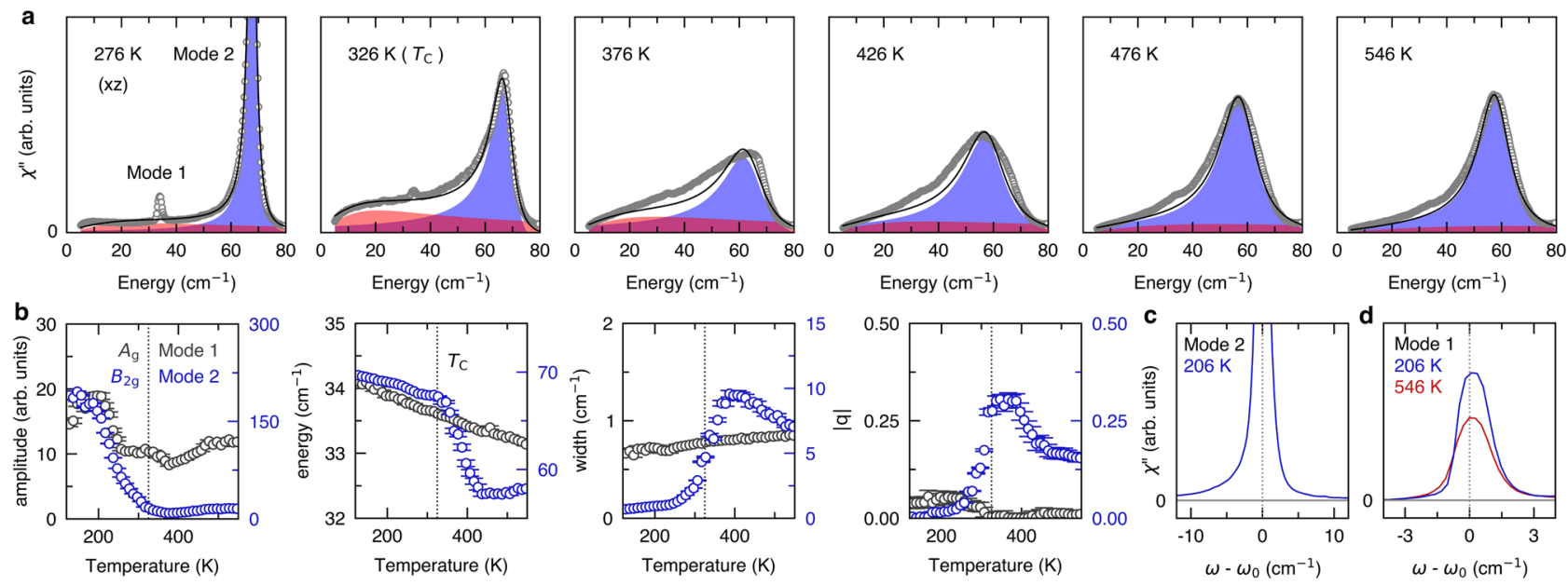

which replaces the previous incorrect version:
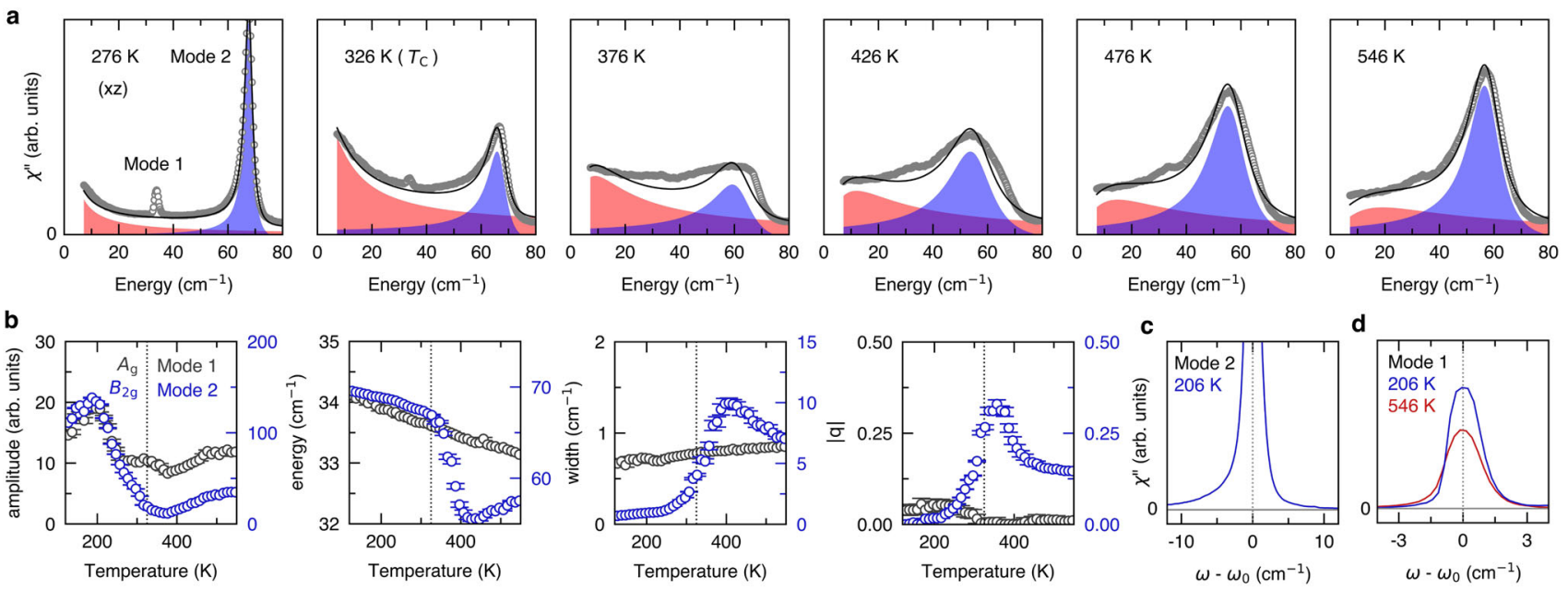
The original version of this Article contained an error in Fig. 4, in which the data was not corrected for the Bose factor.

The correct version is:

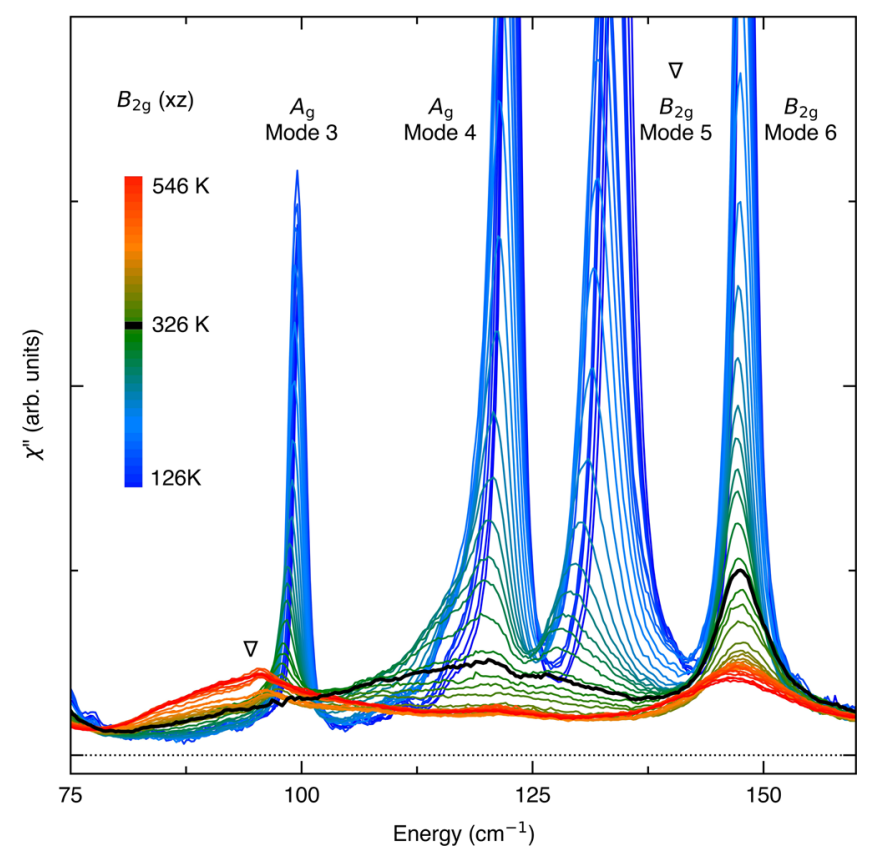

which replaces the previous incorrect version:



The original version of this Article contained an error in the fourth sentence of the Abstract, which incorrectly read "Critical fluctuations of the excitonic order parameter give rise to quasi-elastic scattering of B2g symmetry, whose intensity grows inversely with temperature toward the Weiss temperature of $\mathrm{TW} \approx 237 \mathrm{~K}$, which is arrested by a structural phase transition driven by an acoustic 
phonon of the same symmetry at TC $=325 \mathrm{~K}$ ”. The correct version states " 241 ” in place of " 237 ”.

The first sentence of the sixth paragraph of the Results and discussion originally incorrectly read "The $\chi \mathrm{B} 2 \mathrm{~g}$ shows a Curie-Weiss behavior above TC with the Weiss temperature of $237 \pm 5 \mathrm{~K}$, extracted from linear extrapolation of the inverse susceptibility (Fig. $2 \mathrm{c}$, d)". The correct version states " $241 \pm 9$ ” instead of " $237 \pm 5$ ".

The fourth sentence of the sixth paragraph of the Results and discussion originally incorrectly read "When extrapolated, they intercept the zero frequency at $238 \mathrm{~K}$, giving a self-consistent quantification of the Weiss temperature". The correct version states " 244 " instead of " 238 ".

This has been corrected in both the PDF and HTML versions of the Article.

Published online: 14 May 2021

Open Access This article is licensed under a Creative Commons Attribution 4.0 International License, which permits use, sharing, adaptation, distribution and reproduction in any medium or format, as long as you give appropriate credit to the original author(s) and the source, provide a link to the Creative Commons license, and indicate if changes were made. The images or other third party material in this article are included in the article's Creative Commons license, unless indicated otherwise in a credit line to the material. If material is not included in the article's Creative Commons license and your intended use is not permitted by statutory regulation or exceeds the permitted use, you will need to obtain permission directly from the copyright holder. To view a copy of this license, visit http://creativecommons.org/licenses/by/4.0/.
}

(C) The Author(s) 2021 